\title{
The General Equation of Motion in a Gravitational Field Based Upon the Golden Metric Tensor
}

\author{
Nura Yakubu ${ }^{1}$, Samuel Xede Kofi Howusu ${ }^{2}$, Nuhu Ibrahim ${ }^{3}$, Ado Musa ${ }^{4}$, Abbas Babakura ${ }^{1}$ \\ ${ }^{1}$ Department of Physics, University of Maiduguri, Maiduguri, Nigeria \\ ${ }^{2}$ Theoretical Physics Program, National Mathematics Centre Abuja, Abuja, Nigeria \\ ${ }^{3}$ Department of Physics, Government Science \& Technical College Nguru, Nguru, Nigeria \\ ${ }^{4}$ Department of Physics, Aminu Saleh College of Education Azare Bauchi, Bauchi, Nigeria \\ Email address: \\ nuraphy@gmail.com (N. Yakubu)
}

\section{To cite this article:}

Nura Yakubu, Samuel Xede Kofi Howusu, Nuhu Ibrahim, Ado Musa, Abbas Babakura. The General Equation of Motion in a Gravitational Field Based Upon the Golden Metric Tensor. American Journal of Modern Physics. Vol. 6, No. 6, 2017, pp. 127-131. doi: 10.11648/j.ajmp.20170606.13

Received: August 3, 2017; Accepted: September 4, 2017; Published: September 22, 2017

\begin{abstract}
In this paper, we used Howusu's planetary equation. The general equation of motion is derived for particle of nonzero rest mass in a gravitational field based upon Riemannian geometry and the golden metric tensor which is thereby opens the way for further studies or to pave the way for applications such as planetary theory.
\end{abstract}

Keywords: Golden Metric Tensor, Geodesic Equation, Coefficient of Affine Connection

\section{Introduction}

In the recently published book entitled "The metric tensor for gravitational fields and the mathematical principles of Riemannian theoretical physics", the general equation of motion in the gravitational field based upon Riemannian geometry is given by $[1,2]$

$$
\frac{\mathrm{d}}{\mathrm{d} \tau}\left\{\left(\mathrm{m}_{\mathrm{H}}\right)_{\mathrm{I}} \underline{\mathrm{u}}_{\mathrm{H}}\right\}=\left(\mathrm{m}_{\mathrm{H}}\right)_{\mathrm{P}} \underline{\mathrm{g}}_{\mathrm{H}}
$$

Or equivalently

$$
\left(\mathrm{m}_{\mathrm{H}}\right)_{\mathrm{I}} \underline{\mathrm{a}}_{\mathrm{H}}+\frac{\mathrm{d}}{\mathrm{d} \tau}\left\{\left(\mathrm{m}_{\mathrm{H}}\right)_{\mathrm{I}} \underline{\mathrm{u}}_{\mathrm{H}}\right\}=\left(\mathrm{m}_{\mathrm{H}}\right)_{\mathrm{P}} \underline{\mathrm{g}}_{\mathrm{H}}
$$

Or equivalently

$$
\underline{\mathrm{a}}_{\mathrm{H}}+\frac{1}{\left(\mathrm{~m}_{\mathrm{H}}\right)_{\mathrm{I}}} \frac{\mathrm{d}}{\mathrm{d} \tau}\left\{\left(\mathrm{m}_{\mathrm{H}}\right)_{\mathrm{I}} \underline{\mathrm{u}}_{\mathrm{H}}=\mathrm{g}_{\mathrm{H}}\right.
$$

Where

$\underline{\mathrm{a}}_{\mathrm{H}}$ Riemannian acceleration vector

$\underline{\mathrm{u}}_{\mathrm{H}}$ Riemannian velocity vector

$\underline{g}_{\mathrm{H}}$ Riemannian acceleration due to gravity

$\left(\mathrm{m}_{\mathrm{H}}\right)_{\mathrm{I}}$ General inertial mass

\section{$\left(\mathrm{m}_{\mathrm{H}}\right)_{\mathrm{P}}$ General passive mass}

\section{Method}

In this section we used general equation of motion proposed by Professor Howusu [1, 2] given by

$$
\frac{\mathrm{d}}{\mathrm{d} \tau}\left\{\left(\mathrm{m}_{\mathrm{H}}\right)_{\mathrm{I}} \underline{u}_{\mathrm{H}}\right\}=\left(\mathrm{m}_{\mathrm{H}}\right)_{\mathrm{P}} \underline{\mathrm{g}}_{\mathrm{H}}
$$

Or equivalently

$$
\left(m_{H}\right)_{I} \underline{a}_{H}+\frac{d}{d \tau}\left\{\left(m_{H}\right)_{I} \underline{u}_{H}\right\}=\left(m_{H}\right)_{P} \underline{g}_{H}
$$

Or equivalently

$$
\underline{\mathrm{a}}_{\mathrm{H}}+\frac{1}{\left(\mathrm{~m}_{\mathrm{H}}\right)_{\mathrm{I}}} \frac{\mathrm{d}}{\mathrm{d} \tau}\left\{\left(\mathrm{m}_{\mathrm{H}}\right)_{\mathrm{I}}\right\} \underline{\mathrm{u}}_{\mathrm{H}}=\left(\mathrm{m}_{\mathrm{H}}\right)_{\mathrm{P}} \underline{\mathrm{g}}_{\mathrm{H}}
$$

\subsection{General Linear Acceleration}

We obtained general linear acceleration vector based upon the Riemannian geometry and the golden metric tensor in Spherical polar coordinates proposed by Howusu by replacing the well-known Newton's geodesic equation of motion given as $[1,2]$ 


$$
\begin{gathered}
\frac{\mathrm{d}}{\mathrm{d} \tau}\left(\mathrm{m}_{0} \underline{\mathrm{u}}\right)=\underline{\mathrm{F}}^{\mathrm{g}} \\
\mathrm{m}_{0} \underline{\mathrm{a}}=\underline{\mathrm{F}}^{\mathrm{g}}
\end{gathered}
$$

where

$\mathrm{m}_{0}$ is the rest mass

uis the Euclidean linear velocity

ais the Euclidean linear acceleration with

$\mathrm{F}^{\mathrm{g}}$ is the pure Newton's gravitational force vector

$$
\underline{\mathrm{a}}_{\mathrm{H}}+\frac{1}{\left(\mathrm{~m}_{\mathrm{H}}\right)_{\mathrm{I}}} \frac{\mathrm{d}}{\mathrm{d} \tau}\left\{\left(\mathrm{m}_{\mathrm{H}}\right)_{\mathrm{I}}\right\} \underline{\mathrm{u}}_{\mathrm{H}}=\left(\mathrm{m}_{\mathrm{H}}\right)_{\mathrm{P}} \underline{\mathrm{g}}_{\mathrm{H}}
$$

where the parameters of equation (3) are define as follows $\underline{\mathrm{a}}_{\mathrm{H}}$ is the general linear acceleration vector given as [3]

$$
\left(\mathrm{a}_{\mathrm{H}}\right)_{\mathrm{x}^{1}}=\left(\mathrm{g}_{11}\right)^{\frac{1}{2}}\left\{\begin{array}{c}
\ddot{\mathrm{x}}^{1}+\Gamma_{11}^{1}(\dot{\mathrm{x}})^{2}+\Gamma_{22}^{1}(\dot{\theta})^{2} \\
+\Gamma_{33}^{1}(\dot{\phi})^{2}+\Gamma_{00}^{1}(\dot{\mathrm{x}})^{2} \\
+2 \Gamma_{02}^{1} \dot{\mathrm{x}}^{0} \dot{\theta}+2 \Gamma_{03}^{1} \dot{\mathrm{x}}^{0} \dot{\phi} \\
+2 \Gamma_{12}^{1} \dot{\mathrm{x}} \dot{\theta}+2 \Gamma_{13}^{1} \dot{\mathrm{x}} \dot{\phi} \\
+2 \Gamma_{23}^{1} \dot{\theta} \dot{\phi}+2 \Gamma_{01}^{1} \dot{\mathrm{x}}^{0} \dot{\mathrm{x}}
\end{array}\right\}
$$

$\Gamma_{\alpha \beta}^{\mu}$ is the coefficient of affine connection and is defined by $[4,5$ and 6$]$ :

$$
\Gamma_{\alpha \beta}^{\mu}=\frac{1}{2} g^{\mu \varepsilon}\left(g_{\alpha \varepsilon, \beta}+g_{\varepsilon \beta, \alpha}-g_{\alpha \beta, \varepsilon}\right)
$$

$\left(m_{H}\right)_{I}$ is the general inertial mass given as [7]

$$
\left(\mathrm{m}_{\mathrm{H}}\right)_{\mathrm{I}}=\left[1-\frac{\mathrm{u}^{2}}{\mathrm{c}^{2}}\left(1+\frac{2}{\mathrm{c}^{2}} \mathrm{f}\right)^{-1}\right]^{\frac{1}{2}}\left(1+\frac{2}{\mathrm{c}^{2}} \mathrm{f}\right)^{-\frac{1}{2}} \mathrm{~m}_{0}
$$

$\left(m_{H}\right)_{P}$ is the general passive mass given as

$$
\left(m_{H}\right)_{P}=\left[1-\frac{u^{2}}{c^{2}}\left(1+\frac{2}{c^{2}} f\right)^{-1}\right]^{\frac{1}{2}}\left(1+\frac{2}{c^{2}} f\right)^{-\frac{1}{2}} m_{0}
$$

where $\mathrm{u}$ is the speed of the particle, $\mathrm{f}$ is the gravitational scalar potential, $\mathrm{m}_{0}$ is the zero rest mass of the particle, $c$ is the speed of light.

and $\underline{u}_{H}$ is the general linear velocity defined as

$$
\left(\underline{\mathrm{u}}_{\mathrm{H}}\right)_{\mathrm{x}^{1}}=\left(1+\frac{2}{\mathrm{c}^{2}} \mathrm{f}\right)^{-\frac{1}{2}} \dot{\mathrm{x}}
$$

Similarly for $\left(\underline{u}_{\mathrm{H}}\right)_{\mathrm{x}^{2}},\left(\underline{\mathrm{u}}_{\mathrm{H}}\right)_{\mathrm{x}^{3}},\left(\underline{\mathrm{u}}_{\mathrm{H}}\right)_{\mathrm{x}^{0}}$, where $\mathrm{f}$ is the gravitational scalar potential and $c$ is the speed of light.

\subsection{General Gravitational Intensity (Acceleration Due to Gravity)}

We obtained general gravitational intensity (acceleration due to gravity) vector based upon the Riemannian geometry and the golden metric tensor in Spherical polar coordinates proposed by Howusu by replacing the well-known Newton's gravitational which was built upon the Euclidean gradient operator $[1,2]$

$$
\underline{g}=-\nabla f
$$

where

$f$ is the gravitational scalar potential

$\nabla$ is the Euclidean gradient operator

$\underline{g}$ is the Newton's gravitational intensity (acceleration due to gravity) vector.

with

$$
\begin{aligned}
& g_{r}=-\left(\dot{\mathrm{x}}^{0}\right)^{2} \Gamma_{00}^{1} \\
& \mathrm{~g}_{\theta}=-\left(\dot{\mathrm{x}}^{0}\right)^{2} \Gamma_{00}^{2} \\
& \mathrm{~g}_{\phi}=-\left(\dot{\mathrm{x}}^{0}\right)^{2} \Gamma_{00}^{3} \\
& \mathrm{~g}_{0}=-\left(\dot{\mathrm{x}}^{0}\right)^{2} \Gamma_{00}^{0}
\end{aligned}
$$

where

$\mathrm{g}_{\mu \mathrm{v}}$ is the components of covariant golden metric tensor $\mathrm{g}_{\mathrm{r}}$ is the generalized radial gravitational intensity $\mathrm{g}_{\theta}$ is the generalized polar angle gravitational intensity

$\mathrm{g}_{\phi}$ is the generalized azimuthal angle gravitational intensity

$\mathrm{g}_{0}$ is the generalized time gravitational intensity

$\dot{\mathrm{x}}^{0}$ is the space time velocity and gamma is the coefficient of affine connection.

Consider the motion in a gravitational field exterior to a static homogeneous spherical distribution of mass having radius $R$ and total mass $M$. Then the golden metric tensor in Einstein spherical polar coordinates $\left(r, \theta, \phi, x^{0}\right)$ is given by [8 and 9]

$$
\begin{aligned}
& g_{11}=\left\{1+\frac{2}{c^{2}} f\left(r, \theta, \phi, x^{0}\right)\right\}^{-1} \\
& g_{22}=r^{2}\left\{1+\frac{2}{c^{2}} f\left(r, \theta, \phi, x^{0}\right)\right\}^{-1} \\
& g_{33}=r^{2} \sin ^{2} \theta\left\{1+\frac{2}{c^{2}} f\left(r, \theta, \phi, x^{0}\right)\right\}^{-1} \\
& g_{00}=-\left\{1+\frac{2}{c^{2}} f\left(r, \theta, \phi, x^{0}\right)\right\} \\
& g_{u v}=0, \text { otherwise } \\
& f=f\left(r, \theta, \phi, x^{0}\right)
\end{aligned}
$$

The corresponding Christoffel's symbol of the second kind (or coefficient of affine connection), are given by [10]

$$
\begin{aligned}
& \Gamma_{00}^{1}=\frac{1}{c^{2}}\left(1+\frac{2}{c^{2}} f\right) f_{, 1} \\
& \Gamma_{01}^{1}=\Gamma_{10}^{1}=-\frac{1}{c^{2}}\left(1+\frac{2}{c^{2}} f\right)^{-1} f_{, 0} \\
& \Gamma_{11}^{1}=-\frac{1}{c^{2}}\left(1+\frac{1}{c^{2}} f\right)^{-1} f_{, 1} \\
& \Gamma_{12}^{1}=\Gamma_{21}^{1}=-\frac{1}{c^{2}}\left(1+\frac{2}{c^{2}} f\right)^{-1} f_{, 2}
\end{aligned}
$$




$$
\begin{gathered}
\Gamma_{13}^{1}=\Gamma_{31}^{1}=-\frac{1}{c^{2}}\left(1+\frac{2}{c^{2}} f\right)^{-1} f_{, 3} \\
\Gamma_{22}^{1}=-\frac{r^{2}}{c^{2}}\left(1+\frac{2}{c^{2}} f\right)^{-1} f_{, 1}-r \\
\Gamma_{33}^{1}=\frac{r^{2} \sin ^{2} \theta}{c^{2}}\left(1+\frac{2}{c^{2}} f\right)^{-1} f_{, 1}-r \sin ^{2} \theta
\end{gathered}
$$

and

$$
\begin{gathered}
\Gamma_{00}^{2}=\frac{1}{c^{2} \mathrm{r}^{2}}\left(1+\frac{2}{\mathrm{c}^{2}} \mathrm{f}\right) \mathrm{f}_{, 2} \\
\Gamma_{02}^{2}=\Gamma_{20}^{2}=\frac{1}{\mathrm{c}^{2}}\left(1+\frac{2}{\mathrm{c}^{2}} \mathrm{f}\right)^{-1} \mathrm{f}_{, 0} \\
\Gamma_{11}^{2}=\frac{1}{\mathrm{c}^{2} \mathrm{r}^{2}}\left(1+\frac{1}{\mathrm{c}^{2}} \mathrm{f}\right)^{-1} \mathrm{f}_{, 2} \\
\Gamma_{12}^{2}=\Gamma_{21}^{2}=\frac{1}{\mathrm{r}}-\frac{1}{\mathrm{c}^{2}}\left(1+\frac{2}{\mathrm{c}^{2}} \mathrm{f}\right)^{-1} \mathrm{f}_{, 2} \\
\Gamma_{22}^{2}=-\frac{1}{\mathrm{c}^{2}}\left(1+\frac{2}{\mathrm{c}^{2}} \mathrm{f}\right)^{-1} \mathrm{f}_{, 2} \\
\Gamma_{23}^{2}=\Gamma_{32}^{2}=-\frac{2}{\mathrm{c}^{2}}\left(1+\frac{2}{\mathrm{c}^{2}} \mathrm{f}\right)^{-1} \mathrm{f}_{, 3} \\
\Gamma_{33}^{2}=\frac{\sin ^{2} \theta}{\mathrm{c}^{2}}\left(1+\frac{2}{\mathrm{c}^{2}} \mathrm{f}\right)^{-1} \mathrm{f}_{, 2}-\sin \theta \cos \theta
\end{gathered}
$$

and

$$
\begin{aligned}
& \Gamma_{00}^{3}=\frac{1}{c^{2} r^{2} \sin ^{2} \theta}\left(1+\frac{2}{c^{2}} f\right) f_{, 3} \\
& \Gamma_{03}^{3}=\Gamma_{30}^{3}=\frac{1}{c^{2}}\left(1+\frac{2}{c^{2}} f\right)^{-1} f_{, 3} \\
& \Gamma_{11}^{3}=\frac{1}{c^{2} r^{2} \sin ^{2} \theta}\left(1+\frac{2}{c^{2}} f\right) f_{, 3}
\end{aligned}
$$

$$
\begin{gathered}
\Gamma_{13}^{3}=\Gamma_{31}^{3}=\frac{1}{\mathrm{r}}-\frac{1}{\mathrm{c}^{2}}\left(1+\frac{2}{\mathrm{c}^{2}} \mathrm{f}\right)^{-1} \mathrm{f}_{, 1} \\
\Gamma_{22}^{3}=\frac{1}{\mathrm{c}^{2} \sin ^{2} \theta}\left(1+\frac{2}{\mathrm{c}^{2}} \mathrm{f}\right)^{-1} \mathrm{f}_{, 3} \\
\Gamma_{23}^{3}=\Gamma_{32}^{3}=\cot \theta-\frac{1}{\mathrm{c}^{2}}\left(1+\frac{2}{\mathrm{c}^{2}} \mathrm{f}\right)^{-1} \mathrm{f}_{, 2} \\
\Gamma_{33}^{3}=-\frac{1}{\mathrm{c}^{2}}\left(1+\frac{2}{\mathrm{c}^{2}} \mathrm{f}\right)^{-1} \mathrm{f}_{, 3}
\end{gathered}
$$

and

$$
\begin{aligned}
& \Gamma_{00}^{0}=\frac{1}{c^{2}}\left(1+\frac{2}{c^{2}} f\right)^{-1} f_{, 0} \\
& \Gamma_{01}^{0}=\Gamma_{10}^{0}=\frac{1}{c^{2}}\left(1+\frac{2}{c^{2}} f\right)^{-1} f_{, 1} \\
& \Gamma_{02}^{0}=\Gamma_{20}^{0}=\frac{1}{c^{2}}\left(1+\frac{2}{c^{2}} f\right)^{-1} f_{, 2} \\
& \Gamma_{03}^{0}=\Gamma_{30}^{0}=\frac{1}{c^{2}}\left(1+\frac{2}{c^{2}} f\right)^{-1} f_{, 3} \\
& \Gamma_{01}^{0}=-\frac{1}{c^{2}}\left(1+\frac{2}{c^{2}} f\right)^{-3} f_{, 0} \\
& \Gamma_{22}^{0}=-\frac{r^{2}}{c^{2}}\left(1+\frac{2}{c^{2}} f\right)^{-3} f_{, 0} \\
& \Gamma_{33}^{0}=-\frac{r^{2} \sin ^{2} \theta}{c^{2}}\left(1+\frac{2}{c^{2}} f\right)^{-3} f_{, 0} \\
& \Gamma_{\alpha \beta}^{\mu}=0 ; \text { otherwise }
\end{aligned}
$$

Putting the Christoffel symbols of the second kind into equations (4) we obtain the components of general linear acceleration vector as

$$
\begin{gathered}
\left(\mathrm{a}_{\mathrm{H}}\right)_{\mathrm{r}}=\left(1+\frac{2}{\mathrm{c}^{2}} \mathrm{f}\right)^{-\frac{1}{2}}\left(\ddot{\mathrm{r}}-\mathrm{r} \dot{\theta}^{2}-\mathrm{rsin}^{2} \theta \dot{\phi}\right) \\
+\frac{1}{\mathrm{c}^{2}}\left(1+\frac{2}{\mathrm{c}^{2}} \mathrm{f}\right)^{-\frac{3}{2}}\left[\left\{-\mathrm{f}_{, 1} \dot{\mathrm{r}}^{2}+\mathrm{r}^{2} \mathrm{f}_{, 1} \dot{\theta}^{2}+\mathrm{r}^{2} \sin ^{2} \theta \mathrm{f}_{, 1} \dot{\phi}^{2}-\mathrm{f}_{, 2} \dot{\mathrm{r}} \dot{\theta}-\mathrm{f}_{, 2} \dot{\mathrm{r}} \dot{\phi}\right\}\right] \\
\left(\mathrm{a}_{\mathrm{H}}\right)_{\theta}=\left(1+\frac{2}{\mathrm{c}^{2}} \mathrm{f}\right)^{-\frac{1}{2}}\left(\mathrm{r} \ddot{\theta}+2 \dot{\mathrm{r}} \dot{\theta}-\mathrm{r} \sin \theta \cos \theta \dot{\phi}^{2}\right) \\
+\frac{\mathrm{r}}{\mathrm{c}^{2}}\left(1+\frac{2}{\mathrm{c}^{2}} \mathrm{f}\right)^{-\frac{3}{2}}\left[\left\{\frac{1}{\mathrm{r}^{2}} \mathrm{f}_{, 2} \dot{\mathrm{r}}^{2}-\mathrm{f}_{, 2} \dot{\theta}^{2}+\sin ^{2} \theta \mathrm{f}_{, 2} \dot{\phi}^{2}-2 \mathrm{f}_{, 1} \dot{\mathrm{r}} \dot{\theta}-2 \mathrm{f}_{, 3} \dot{\theta} \dot{\phi}\right\}\right] \\
+\frac{\mathrm{r} \sin \theta}{\mathrm{c}^{2}}\left(1+\frac{2}{\mathrm{c}^{2}} \mathrm{f}\right)_{\phi}=\sin ^{-\frac{3}{2}}\left[\left\{\frac{1}{\mathrm{r}^{2} \sin ^{2}} \mathrm{f}_{, 3} \dot{\mathrm{r}}+\frac{1}{\mathrm{sin}^{2}} \mathrm{f}_{, 3} \dot{\theta}^{2}-\mathrm{f}_{, 1} \dot{\mathrm{r}} \dot{\phi}-\mathrm{f}_{, 2} \dot{\theta} \dot{\phi}\right\}\right] \\
\left(\mathrm{a}_{\mathrm{H}}\right)_{\mathrm{x}^{0}}=i\left(1+\frac{2}{\mathrm{c}^{2}} \mathrm{f}\right)^{-\frac{1}{2}}(\mathrm{r} \ddot{\phi}+\dot{\mathrm{r}} \dot{\phi}+\mathrm{r} \cot \theta \dot{\theta} \dot{\phi}) \\
-\frac{i}{\mathrm{c}^{2}}\left(1+\frac{2}{\mathrm{c}^{2}} \mathrm{f}\right)^{\frac{1}{2}}\left[\left\{\left(1+\frac{2}{\mathrm{c}^{2}} \mathrm{f}\right)^{-2} \mathrm{f}_{, 0} \dot{\mathrm{r}}^{2}+\left(1+\frac{2}{\mathrm{c}^{2}} \mathrm{f}\right)^{-2} \mathrm{f}_{, 0} \dot{\theta}^{2}+\mathrm{f}_{, 3} \dot{\mathrm{x}}^{0} \dot{\phi}+\mathrm{f}_{, 2} \dot{\mathrm{x}}^{0} \dot{\theta}+\mathrm{f}_{, 1} \dot{\mathrm{x}}^{0} \dot{\mathrm{r}}\right\}\right]
\end{gathered}
$$

Putting the Christoffel symbols of the second kind into equation (7) to (10) we obtain the component of the general 
gravitational intensity (or acceleration due to gravity) vector as

$$
\begin{aligned}
& \left(g_{H}\right)_{r}=-\dot{t}^{2}\left(1+\frac{2}{c^{2}} f\right)^{\frac{1}{2}} f_{, 1} \\
& \left(g_{H}\right)_{\theta}=-\dot{t}^{2} \cdot \frac{1}{r}\left(1+\frac{2}{c^{2}} f\right)^{\frac{1}{2}} f_{, 2} \\
& \left(g_{H}\right)_{\phi}=-\dot{t}^{2} \cdot \frac{1}{r \sin \theta}\left(1+\frac{2}{c^{2}} f\right)^{\frac{1}{2}} f_{, 3} \\
& \left(g_{H}\right)_{x^{0}}=i \dot{t}^{2}\left(1+\frac{2}{c^{2}} f\right)^{\frac{1}{2}} f_{, 0}
\end{aligned}
$$

and also we obtain the component of the general linear velocity vector as

$$
\mathrm{u}_{\mathrm{r}}=\left(1+\frac{2}{\mathrm{c}^{2}} \mathrm{f}\right)^{-\frac{1}{2}} \dot{\mathrm{r}}
$$

$$
\begin{gathered}
\mathrm{u}_{\theta}=\mathrm{r}\left(1+\frac{2}{\mathrm{c}^{2}} \mathrm{f}\right)^{-\frac{1}{2}} \dot{\theta} \\
\mathrm{u}_{\phi}=\mathrm{r} \sin \theta\left(1+\frac{2}{\mathrm{c}^{2}} \mathrm{f}\right)^{-\frac{1}{2}} \dot{\phi} \\
\mathrm{u}_{0}=i\left(1+\frac{2}{\mathrm{c}^{2}} \mathrm{f}\right)^{-\frac{1}{2}} \mathrm{ct}
\end{gathered}
$$

\section{Results}

Finally substituting equations (45) to (48), (49) to (52) and (53) to (56) into equation (3) we obtain the general equation of motion for a particle of non-zero rest mass in a gravitational field based upon the Riemannian geometry and the golden metric tensor as

$$
\begin{aligned}
& \left(1+\frac{2}{c^{2}} f\right)^{-\frac{1}{2}}\left(\ddot{r}-r \dot{\theta}^{2}-r \sin ^{2} \theta \dot{\phi}\right) \\
& +\frac{1}{c^{2}}\left(1+\frac{2}{c^{2}} f\right)^{-\frac{3}{2}}\left[-f_{, 1} \dot{\mathrm{r}}^{2}+\mathrm{r}^{2} \mathrm{f}_{, 1} \dot{\theta}^{2}+\mathrm{r}^{2} \sin ^{2} \theta \mathrm{f}_{, 1} \dot{\phi}^{2}-\mathrm{f}_{, 2} \dot{\mathrm{r}} \dot{\theta}-\mathrm{f}_{, 2} \dot{\mathrm{r}} \dot{\phi}\right] \\
& +\frac{1}{\left(m_{\mathrm{H}}\right)_{\mathrm{I}}}\left\{\frac{\mathrm{d}}{\mathrm{d} \tau}\left(\mathrm{m}_{\mathrm{H}}\right)_{\mathrm{I}}\right\}\left(1+\frac{2}{\mathrm{c}^{2}} \mathrm{f}\right)^{-\frac{1}{2}} \dot{\mathrm{r}}=-\dot{\mathrm{t}}^{2}\left(1+\frac{2}{\mathrm{c}^{2}} \mathrm{f}\right)^{\frac{1}{2}} \mathrm{f}_{, 1} \\
& \left(1+\frac{2}{c^{2}} f\right)^{-\frac{1}{2}}\left(r \ddot{\theta}+2 \dot{r} \dot{\theta}-r \sin \theta \cos \theta \dot{\phi}^{2}\right) \\
& +\frac{1}{c^{2}}\left(1+\frac{2}{c^{2}} f\right)^{-\frac{3}{2}}\left[\frac{1}{r^{2}} f_{, 2} \dot{r}^{2}-f_{, 2} \dot{\theta}^{2}+\sin ^{2} \theta f_{, 2} \dot{\phi}^{2}-2 f_{, 1} \dot{\mathrm{r}} \dot{\theta}-2 f_{, 3} \dot{\theta} \dot{\phi}\right] \\
& +\frac{1}{\left(\mathrm{~m}_{\mathrm{H}}\right)_{\mathrm{I}}}\left\{\frac{\mathrm{d}}{\mathrm{d} \tau}\left(\mathrm{m}_{\mathrm{H}}\right)_{\mathrm{I}}\right\}\left(1+\frac{2}{\mathrm{c}^{2}} \mathrm{f}\right)^{-\frac{1}{2}} \dot{\theta}=-\dot{\mathrm{t}}^{2} \cdot \frac{1}{\mathrm{r}}\left(1+\frac{2}{\mathrm{c}^{2}} \mathrm{f}\right)^{\frac{1}{2}} \mathrm{f}_{, 2} \\
& \sin \theta\left(1+\frac{2}{c^{2}} f\right)^{-\frac{1}{2}}(r \ddot{\phi}+\dot{r} \dot{\phi}+r \cot \theta \dot{\theta} \dot{\phi}) \\
& +\frac{\mathrm{r} \sin \theta}{\mathrm{c}^{2}}\left(1+\frac{2}{\mathrm{c}^{2}} \mathrm{f}\right)^{-\frac{3}{2}}\left[\frac{1}{\mathrm{r}^{2} \sin ^{2}} \mathrm{f}_{, 3} \dot{\mathrm{r}}+\frac{1}{\sin ^{2}} \mathrm{f}_{3} \dot{\theta}^{2}-\mathrm{f}_{, 1} \dot{\mathrm{r}} \dot{\phi}-\mathrm{f}_{, 2} \dot{\theta} \dot{\phi}\right] \\
& +\frac{1}{\left(m_{H}\right)_{I}}\left\{\frac{d}{d \tau}\left(m_{H}\right)_{I}\right\}\left(1+\frac{2}{c^{2}} f\right)^{-\frac{1}{2}} \dot{\phi}=-\dot{t}^{2} \cdot \frac{1}{r \sin \theta}\left(1+\frac{2}{c^{2}} f^{\frac{1}{2}} f_{, 3}\right. \\
& \left(1+\frac{2}{c^{2}} f\right)^{-\frac{1}{2}} \ddot{x}^{0} \\
& -\frac{i}{\mathrm{c}^{2}}\left(1+\frac{2}{\mathrm{c}^{2}} \mathrm{f}\right)^{\frac{1}{2}}\left[\left\{\left(1+\frac{2}{\mathrm{c}^{2}} \mathrm{f}\right)^{-2} \mathrm{f}_{, 0} \dot{\mathrm{r}}^{2}+\left(1+\frac{2}{\mathrm{c}^{2}} \mathrm{f}\right)^{-2} \mathrm{f}_{, 0} \dot{\theta}^{2}+\mathrm{f}_{, 3} \dot{\mathrm{x}}^{0} \dot{\phi}+\mathrm{f}_{, 2} \dot{\mathrm{x}}^{0} \dot{\theta}+\mathrm{f}_{, 1} \dot{\mathrm{x}}^{0} \dot{\mathrm{r}}\right\}\right] \\
& +\frac{1}{\left(\mathrm{~m}_{\mathrm{H}}\right)_{\mathrm{I}}}\left\{\frac{\mathrm{d}}{\mathrm{d} \tau}\left(\mathrm{m}_{\mathrm{H}}\right)_{\mathrm{I}}\right\}\left(1+\frac{2}{\mathrm{c}^{2}} \mathrm{f}\right)^{-\frac{1}{2}} \mathrm{ct}=\dot{\mathrm{t}}^{2}\left(1+\frac{2}{\mathrm{c}^{2}} \mathrm{f}\right)^{\frac{1}{2}} \mathrm{f}_{, 0}
\end{aligned}
$$

\section{Discussion}

Note that equations (57) to (60) obtained in this paper are now available for both physicists and mathematicians alike to obtain corresponding revisions to the planetary equation of motion and hence planetary parameters such as the anomalous orbital precession in the solar system, orbital eccentricity, orbital period, perihelion distance and aphelion 
distance.

\section{Conclusion}

In this paper we succeed in obtaining equation (45) - (48) the components of the general linear acceleration vector. Also in this paper we obtained the components of the general gravitational intensity (or acceleration due to gravity) that are equation (49) - (52). Also obtained in this paper are general equations of motion that is equations (57) - (60). The results obtained in this paper are now available for both physicists and mathematicians alike to apply them in solving planetary problems based upon Riemannian geometry.

\section{References}

[1] Howusu, S. X. K. (2009). "Riemannian Revolutions in mathematics and physics II The great metric tensor and principles". Jos University Press

[2] Howusu, S. X. K. (2009). "Riemannian Revolutions in physics and mathematics $\mathrm{V}$ the golden metric tensor in orthogonal curvilinear coordinates". Pp. (1-9)

[3] Weinberg S. (1972). Principles and applications of the General Theory of Relativity. New York: J. Wiley and Sons
[4] Rajput, B. S. (2010). "Mathematical physics. Pragati Prakashan publisher." Pp (891-895)

[5] Spiegel, M. R. (1974) "Theory and problems of vector analysis and introduction to tensor analysis". McGraw Hill, New York. Pp (193-195)5-7

[6] Howusu, S. X. K. (2009). "Riemannian Revolution in Physics and Mathematics II; The Great Metric Tensor and Principles". Pp. (40-41)

[7] N. Yakubu, S. X. K. Howusu, W. L. Lumbi, N. Ibrahim. (2016): Solution of Newton's Gravitational Field Equation of a Static Homogeneous Oblate Spheroidal Massive Body: International Journal of Theoretical and Mathematical Physics. 6(3): 104-109

[8] Y. Nura, S. X. K. Howusu, L. W. Lumbi, I. Nuhu, A. Hayatu.(2017). The Generalized Planetary Equations Based upon Riemannian geometry and the Golden Metric Tensor: International Journal of Theoretical and Mathematical Physics, 7(2): 25-35 DOI: 10.5923/j.ijtmp.20170702.02

[9] Nura, Y. Howusu S. X. K. and Nuhu, I. (2016). General linear acceleration vector based on the golden metric tensor in Spherical polar coordinates (paper I): International Journal of Current Research in Life Sciences Vol. 05, No. 12, pp. 627-633

[10] Howusu S. X. K. (2009). The metric tensors for gravitational Fields and the mathematical principles of Riemannian theoretical physics. Jos University Press. Pp 15-16 